\title{
Properties of resonantly produced sterile neutrino dark matter subhaloes
}

\author{
Shunsaku Horiuchi, ${ }^{1 \star}$ Brandon Bozek, ${ }^{2 \star}$ Kevork N. Abazajian, ${ }^{3}$ \\ Michael Boylan-Kolchin, ${ }^{2}$ James S. Bullock, ${ }^{3}$ Shea Garrison-Kimmel ${ }^{4}$ \\ and Jose Onorbe ${ }^{5}$ \\ ${ }^{1}$ Center for Neutrino Physics, Department of Physics, Virginia Tech, 850 W Campus Drive, Blacksburg, VA 24061, USA \\ ${ }^{2}$ Department of Astronomy, The University of Texas at Austin, 2515 Speedway, Stop C1400, Austin, TX 78712, USA \\ ${ }^{3}$ Center for Cosmology, Department of Physics and Astronomy, University of California, Irvine, CA 92697, USA \\ ${ }^{4}$ TAPIR, California Institute of Technology, Pasadena, CA 91125, USA \\ ${ }^{5}$ Max-Planck-Institut fuer Astronomie, Koenigstuhl 17, D-69117 Heidelberg, Germany
}

Accepted 2015 December 10. Received 2015 December 10; in original form 2015 October 28

\begin{abstract}
The anomalous $3.55 \mathrm{keV} \mathrm{X}$-ray line recently detected towards a number of massive dark matter objects may be interpreted as the radiative decays of $7.1 \mathrm{keV}$ mass sterile neutrino dark matter. Depending on its parameters, the sterile neutrino can range from cold to warm dark matter with small-scale suppression that differs in form from commonly adopted thermal warm dark matter. Here, we numerically investigate the subhalo properties for $7.1 \mathrm{keV}$ sterile neutrino dark matter produced via the resonant Shi-Fuller mechanism. Using accurate matter power spectra, we run cosmological zoom-in simulations of a Milky Way-sized halo and explore the abundance of massive subhaloes, their radial distributions, and their internal structure. We also simulate the halo with thermal $2.0 \mathrm{keV}$ warm dark matter for comparison and discuss quantitative differences. We find that the resonantly produced sterile neutrino model for the $3.55 \mathrm{keV}$ line provides a good description of structures in the Local Group, including the number of satellite dwarf galaxies and their radial distribution, and largely mitigates the too-big-to-fail problem. Future searches for satellite galaxies by deep surveys, such as the Dark Energy Survey, Large Synoptic Survey Telescope, and Wide Field Infrared Survey Telescope, will be a strong direct test of warm dark matter scenarios.
\end{abstract}

Key words: Galaxy: halo-Local Group-cosmology: theory-dark matter.

\section{INTRODUCTION}

The Lambda cold dark matter $(\Lambda \mathrm{CDM})$ paradigm has been extremely successful in explaining a variety of observations on cosmological scales (e.g. Ho et al. 2012; Hinshaw et al. 2013; Planck Collaboration XXIV 2015). Tests on galactic and subgalactic scales, while often complicated by the physics of galaxy formation, provide crucial verification of the $\Lambda$ CDM model. Several issues with $\Lambda \mathrm{CDM}$ on small scales have been highlighted in the past decade, prompting investigations into galaxy formation within the $\Lambda \mathrm{CDM}$ model, as well as alternative models of dark matter.

Among the earliest and most prominent small-scale issue is the 'missing satellites' problem: the large excess in the number of expected satellite galaxies of the Milky Way (MW), which outnumber those observed by a factor of 10 or more (Klypin et al. 1999; Moore et al. 1999). Another issue is the core/cusp problem, which is seen in, e.g. the flatter central dark matter density profiles observed in many low-surface brightness (LSB) galaxies (e.g. Simon et al. 2005;

^E-mail: horiuchi@vt.edu (SH); bozek@astro.as.utexas.edu (BB)
Donato et al. 2009; Oh et al. 2011) when compared with the cuspy profiles predicted by pure $\Lambda$ CDM simulations (Flores \& Primack 1994; Navarro, Frenk \& White 1997). More recently, the most massive subhaloes of MW-sized hosts have been shown to contain too much dark matter to accommodate the stellar kinematic data of the observed MW dwarf spheroidal (dSph) satellite galaxies (BoylanKolchin, Bullock \& Kaplinghat 2011, 2012). The number of massive subhaloes failing in this way ('massive failures') is typically $\sim 20$, depending on the halo mass, cosmology, and other variables (e.g. Garrison-Kimmel et al. 2014b; Griffen et al. 2015), and constitute the 'too-big-to-fail' (TBTF) problem.

Whether these small-scale issues motivate a change to the $\Lambda \mathrm{CDM}$ paradigm depends quantitatively on the degree to which feedback and other baryonic processes can remedy the discrepancies. For example, studies have postulated how galaxy formation does not follow the subhalo mass at $z=0$, but rather, the maximum mass at some earlier epoch, often at reionization. This reduces the number of expected satellites and helps to remedy the missing satellites problem (Bullock, Kravtsov \& Weinberg 2000; Kravtsov, Gnedin \& Klypin 2004; Ricotti \& Gnedin 2005; Koposov et al. 2009; Okamoto \& Frenk 2009; Busha et al. 2010). Simultaneously, searches with 
the Sloan Digital Sky Survey (SDSS) has revealed some dozen more dSph galaxies (Belokurov et al. 2007), and searches in the Southern hemisphere are starting to produce results in the same direction (DES Collaboration 2015; Drlica-Wagner et al. 2015; Koposov et al. 2015). Studies have also revealed the gross incompleteness of current satellite samples due to a combination of incomplete sky coverage, luminosity bias, and surface brightness limits (Tollerud et al. 2008; Walsh, Willman \& Jerjen 2009; Hargis, Willman \& Peter 2014).

Concerning the inner dark matter densities, baryonic processes can effectively reduce the central dark matter densities of LSB host haloes, addressing the core/cusp problem (e.g. Governato et al. 2012; Pontzen \& Governato 2012; Di Cintio et al. 2014; Del Popolo \& Pace 2015; Oñorbe et al. 2015; Simpson et al. 2015). However, Peñarrubia et al. (2012) and Garrison-Kimmel et al. (2013) have argued that these effects are insufficient to affect dimmer satellites relevant for TBTF (but, see Gritschneder \& Lin 2013; Amorisco, Zavala \& de Boer 2014). On the other hand, environmental effects, coupled with baryonic interactions, can be conducive to more efficient inner density reduction even on these scales (Zolotov et al. 2012; Arraki et al. 2014; Brooks \& Zolotov 2014; Del Popolo et al. 2014; Brook \& Di Cintio 2015; Oñorbe et al. 2015). However, the TBTF issue continues to be observed out in field galaxies of the Local Group, where environmental effects are greatly reduced or non-existent (Ferrero et al. 2012; Garrison-Kimmel et al. 2014b; Papastergis et al. 2015). Therefore, although many plausible mechanisms within the context of $\Lambda \mathrm{CDM}$ exist to explain small-scale issues, it is not clear if they are where the solution lies.

Various alternatives to $\Lambda \mathrm{CDM}$ have been investigated in order to resolve small-scales issues, including non-standard primordial power spectra (Kamionkowski \& Liddle 2000; Zentner \& Bullock 2002; Garrison-Kimmel et al. 2014c; Polisensky \& Ricotti 2014) and modifications to CDM, e.g. self-interacting dark matter (Vogelsberger, Zavala \& Loeb 2012; Rocha et al. 2013; Vogelsberger et al. 2014; Elbert et al. 2015), warm dark matter (WDM; Anderhalden et al. 2012, 2013; Lovell et al. 2012, 2014; Schneider, Smith \& Reed 2013; Horiuchi et al. 2014; Schneider et al. 2014; Reed et al. 2015), decaying dark matter (e.g. Wang et al. 2014), and axion dark matter (e.g. Marsh \& Pop 2015).

From a particle physics perspective, sterile neutrinos of $\sim \mathrm{keV}$ mass provide a compelling WDM candidate. Produced in the Early Universe by oscillations with the active neutrino, ${ }^{1}$ the sterile neutrino is able to generate dark matter of various warmness (or more precisely, suppression in the primordial matter power spectrum) depending on its mass and production mechanism (e.g. Abazajian, Fuller \& Patel 2001a). The same mixing responsible for production leads to sterile neutrino decay, which opens a search strategy using X-ray observations from nearby massive dark matter objects (Abazajian, Fuller \& Tucker 2001b).

Recently, excess X-ray flux have been observed at $\sim 3.55 \mathrm{keV}$ from observations of stacked galaxy clusters (Bulbul et al. 2014b), Perseus (Boyarsky et al. 2014a; Bulbul et al. 2014b), M31 (Boyarsky et al. 2014a), the MW (Boyarsky et al. 2014b), and eight additional galaxy clusters (Iakubovskyi et al. 2015). There is ongoing active debate regarding the significance and interpretation of this signal (Bulbul et al. 2014a; Jeltema \& Profumo 2014, 2015; Malyshev, Neronov \& Eckert 2014; Riemer-Sorensen 2014; Ander-

\footnotetext{
${ }^{1}$ Sterile neutrinos produced in the decays of other heavier relics, e.g. Kusenko (2006) and Shaposhnikov \& Tkachev (2006), typically generate CDM.
}
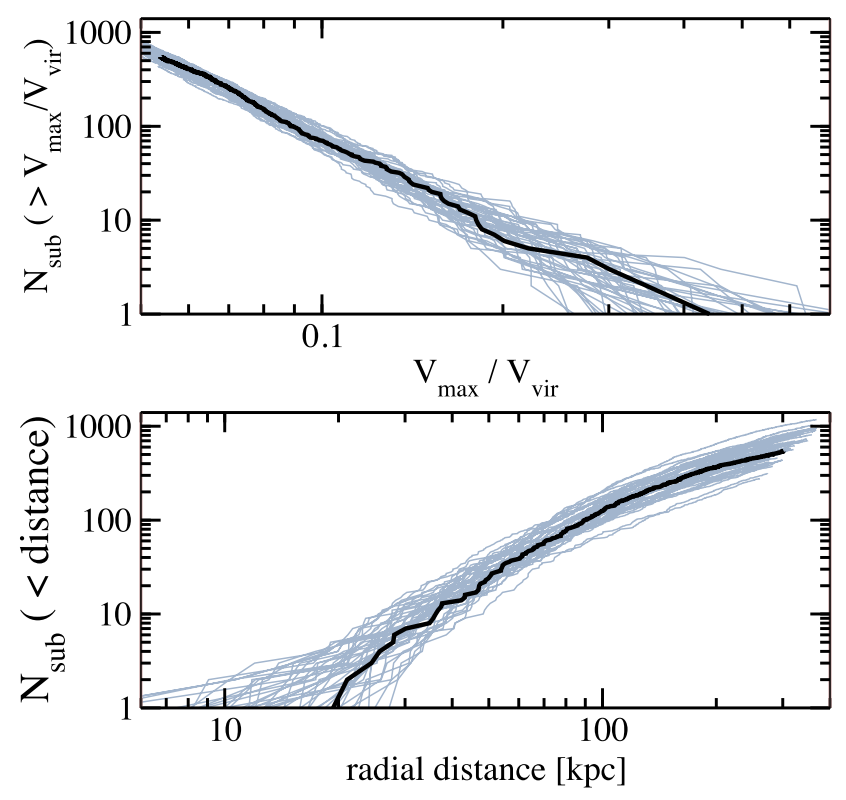

Figure 1. Distributions of subhalo $V_{\max } / V_{\text {vir }}$ (top panel) and radial distance (bottom panel) for the haloes simulated in the ELVIS suite (grey). The particular halo used in this study for WDM simulations is highlighted by the thick solid line, indicating its central behaviour among the CDM realizations of the ELVIS suite.

son, Churazov \& Bregman 2015; Iakubovskyi et al. 2015; Tamura et al. 2015; Urban et al. 2015). Interpreted as a sterile neutrino decay signal (Abazajian 2014), the simplest scenario is a $\sim 7.1 \mathrm{keV}$ sterile neutrino produced by the Shi-Fuller resonantly enhanced mixing mechanism (Shi \& Fuller 1999).

The focus of this paper is to quantitatively explore the subhalo properties in a $7.1 \mathrm{keV}$ sterile neutrino cosmology using dissipationless simulations. We adopt the specific sterile neutrino parameters suggested by the anomalous $3.55 \mathrm{keV}$ line measurement of Bulbul et al. (2014b) and Boyarsky et al. (2014a). More generally, however, our attempt is to simulate the accurate formation of structure in the Shi-Fuller resonant sterile neutrino scenario. In particular, the resonant production of sterile neutrinos has recently been reinvestigated by Venumadhav et al. (2015). By including previously neglected effects of the redistribution of lepton asymmetry and the neutrino opacity, as well as a more accurate treatment of the scattering rates through the quark-hadron transition, the authors provide accurate sterile neutrino phase-space densities. Using these updated distributions as inputs, we run dark matter only collisionless $N$-body simulations, which allows us to explore the implications for Local Group satellite counts and their internal kinematics, specifically addressing the missing satellites and TBTF problems.

The paper is organized as follows. In Section 2, we summarize our simulation and analysis methods. In Section 3, we present our results, including satellite counts in Section 3.1, satellite radial distribution in Section 3.2, and internal structure (implications for TBTF) in Section 3.3. Finally, we present discussions and conclusions in Section 4.

\section{SIMULATIONS}

We run collisionless dark matter-only simulations with the publicly available Tree-PM $N$-body simulation code GADGET- $2^{2}$ (Springel

\footnotetext{
${ }^{2}$ http://www.mpa-garching.mpg.de/gadget/
} 


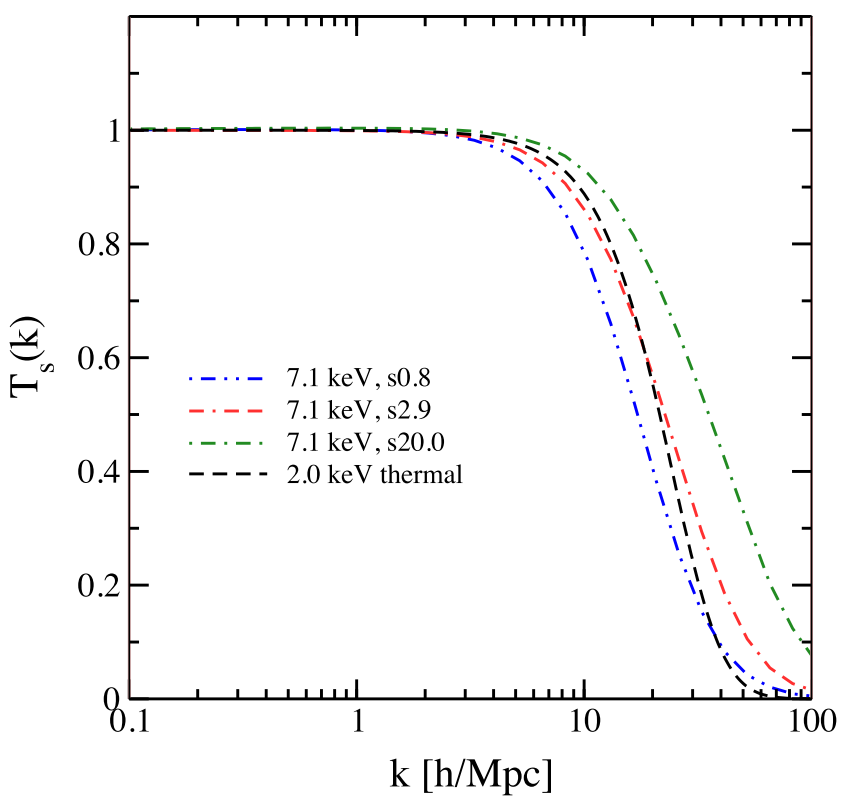

Figure 2. Square root of the sterile neutrino power spectrum relative to $\mathrm{CDM}, T_{s}(k)=\sqrt{P_{s}(k) / P_{\mathrm{CDM}}(k)}$. Shown are three resonantly produced sterile neutrino cases, $\mathrm{s} 0.8$ (blue double-dot-dashed), s2.9 (red dot-doubledashed), and s20.0 (green dot-dashed). For comparison, a $2.0 \mathrm{keV}$ thermal WDM is shown (black dashed). The initial cutoff shape is similar between the $2.0 \mathrm{keV}$ thermal and s2.9 resonant cases, but the tails show quantitative differences.

2005). We present four zoom-in simulations in WDM, all with Wilkinson Microwave Anisotropy Probe 7 cosmological parameters: $\sigma_{8}=0.801, \Omega_{\mathrm{m}}=0.266, \Omega_{\mathrm{b}}=0.0449, \Omega_{\Lambda}=0.734, n_{\mathrm{s}}=0.963$, and $h=0.71$ (Larson et al. 2011). Simulations were initialized at redshift 125 with initial conditions selected from the Exploring the Local Volume In Simulations project (ELVIS; ${ }^{3}$ Garrison-Kimmel et al. 2014a) and created with MUSIC ${ }^{4}$ (Hahn \& Abel 2011). ELVIS is a suite of $48 \Lambda \mathrm{CDM}$ zoom-in simulations designed to study the Local Group. It consists of 24 haloes in paired systems that are chosen to resemble the MW and M31 in mass, relative kinematics, and environment, as well as an additional 24 haloes that are isolated mass-matched analogues. Lagrange volumes are determined by all particles within $5 R_{\text {vir }}$ of the halo centre in the final timestep for isolated analogues, and $4 R_{\mathrm{vir}}$ of either MW or M31 hosts for pairs. We refer the reader to Oñorbe et al. (2014) and Garrison-Kimmel et al. (2014a) for the detail methodology of the zoom-in simulations and ELVIS, respectively.

We select from the ELVIS suite one halo that is closest to the average over the ELVIS suite in two key subhalo properties - the subhalo $V_{\max }$ and radial position distributions. Here, the circular velocity is $V_{\text {circ }}=\sqrt{G M(<r) / r}$, and its peak circular velocity and the radius at which it peaks are denoted as $V_{\max }$ and $R_{\max }$, respectively. Fig. 1 shows our adopted halo in thick solid black, compared to the entire ELVIS suite (grey solid).

To build the WDM initial power spectra, CDM transfer functions were first generated using the publicly available $\mathrm{CAMB}^{5}$ cosmic microwave background boltzmann code (Lewis, Challinor \& Lasenby

\footnotetext{
${ }^{3}$ http://localgroup.ps.uci.edu/elvis/index.html

${ }^{4}$ http://www.phys.ethz.ch/hahn/MUSIC/

5 http://camb.info/
}

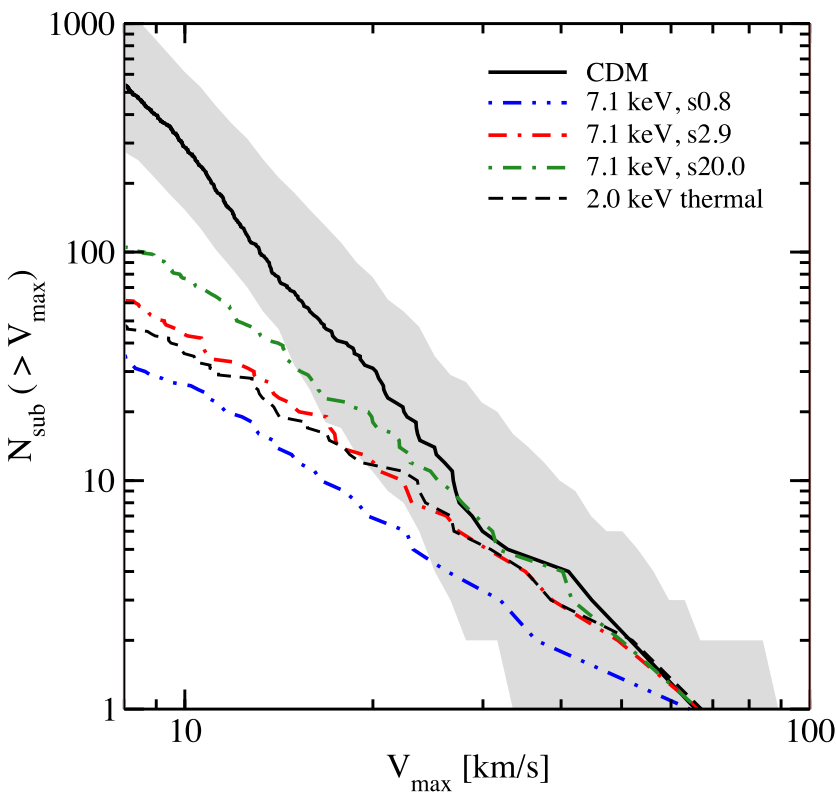

Figure 3. Cumulative distributions of subhaloes within the virial radius in $V_{\max }$. Only subhaloes with $V_{\max }>8 \mathrm{~km} \mathrm{~s}^{-1}$ are shown, due to resolution as well as potential contamination from spurious subhaloes at lower $V_{\max }$. Shown are resonantly generated sterile neutrinos s0.8 (blue double-dotdashed), s2.9 (red dot-double-dashed), and s20.0 (green dot-dashed), a $2.0 \mathrm{keV}$ thermal WDM (black dashed), and CDM (black solid), as labelled. For the CDM, the grey band indicates the scatter determined from the ELVIS suite of simulations.

2000). These were modified according to the resonant sterile neutrino production calculations of Venumadhav et al. (2015), which include effects of the redistribution of lepton asymmetry and the neutrino opacity, as well as a more detail treatment during the quarkhadron transition. These affect the primordial momentum distributions of the sterile neutrino, which in turn quantitatively change the matter power spectrum. Fig. 2 shows the square root of the suppression of the power spectrum, $T_{s}(k)=\sqrt{P_{s}(k) / P_{\mathrm{CDM}}(k)}$, for the three sterile neutrino parameters studied in this paper: all have masses of $m_{s}=7.1 \mathrm{keV}$, but different mixing angles $\sin ^{2} 2 \theta=0.800 \times 10^{-11}$ (labelled s0.8), $2.899 \times 10^{-11}$ (labelled s2.9), and $20.000 \times 10^{-11}$ (labelled s20.0). The lepton asymmetries required to reproduce the observed dark matter abundance $\Omega_{\mathrm{dm}}$ are $\approx(13.0-13.1) \times 10^{-5}$, $(8.32-8.39) \times 10^{-5}$, and $(6.7-6.8) \times 10^{-5}$, respectively. ${ }^{6}$ Also shown for comparison is the transfer function for a thermal WDM based on Bode, Ostriker \& Turok (2001). Specifically, we use the functional forms in their appendix with values adjusted to our cosmology, and fix the mass to a thermal $2.0 \mathrm{keV}$ WDM particle. The $2.0 \mathrm{keV}$ thermal particle was chosen due to its close resemblance to the s2.9 sterile neutrino, thus serving as a good comparison; it was also identified as being near the centre of the range of WDM cutoff scales for the $7.1 \mathrm{keV}$ resonant sterile neutrino in Abazajian (2014). A companion work by Bozek et al. (2015) simulates a MW/M31 pair from the ELVIS suite in a variety of sterile neutrino models (including the s2.9 model) to examine the impact of these models in the Local Group environment.

All simulations were run with a $z=0$ Plummer equivalent force softening of $141 \mathrm{pc}$ in the highest resolution zoom-in

\footnotetext{
${ }^{6}$ These are based on Planck cosmology with $\Omega_{\mathrm{dm}} h^{2}=0.119$, but the cosmology dependence is weak (Abazajian et al. 2001b).
} 
Table 1. Properties of the main halo: virial mass $M_{\mathrm{vir}}$, virial radius $R_{\mathrm{vir}}$, circular velocity $V_{\mathrm{vir}}$, peak of the circular velocity $V_{\max }$, and the radius of peak circular velocity $R_{\max }$. The last three columns show the number of subhaloes within the virial radius with $V_{\max }>8 \mathrm{~km} \mathrm{~s}^{-1}, V_{\max }>15 \mathrm{~km} \mathrm{~s}^{-1}$, and $V_{\text {peak }}>20 \mathrm{~km} \mathrm{~s}{ }^{-1}$, respectively.

\begin{tabular}{lcccccccc}
\hline & $\begin{array}{c}M_{\text {vir }} \\
\left(10^{12} \mathrm{M}_{\odot}\right)\end{array}$ & $\begin{array}{c}R_{\text {vir }} \\
(\mathrm{kpc})\end{array}$ & $\begin{array}{c}V_{\text {vir }} \\
\left(\mathrm{km} \mathrm{s}^{-1}\right)\end{array}$ & $\begin{array}{c}V_{\max } \\
\left(\mathrm{km} \mathrm{s}^{-1}\right)\end{array}$ & $\begin{array}{c}R_{\max } \\
(\mathrm{kpc})\end{array}$ & $\begin{array}{c}\text { Number of subhaloes } \\
\left(V_{\max }>8 \mathrm{~km} \mathrm{~s}^{-1}\right)\end{array}$ & $\begin{array}{c}\text { Number of subhaloes } \\
\left(V_{\max }>15 \mathrm{~km} \mathrm{~s}^{-1}\right)\end{array}$ & $\begin{array}{c}\text { Number of subhaloes } \\
\left(V_{\text {peak }}>20 \mathrm{~km} \mathrm{~s}\right.\end{array}$ \\
\hline CDM & 1.56 & 302 & 149 & 179 & 47.7 & 540 & 66 \\
WDM_s0.8 & 1.54 & 301 & 148 & 180 & 46.0 & 35 & 12 & 20 \\
WDM_s2.9 & 1.55 & 302 & 149 & 180 & 46.4 & 61 & 31 & 23 \\
WDM_s20.0 & 1.55 & 301 & 149 & 180 & 45.8 & 105 & 38 \\
WDM_2.0keV & 1.55 & 301 & 149 & 179 & 48.4 & 48 & 23 \\
\hline
\end{tabular}

region, which contains particles of mass $1.9 \times 10^{5} \mathrm{M}_{\odot}$. Resolution tests performed in Garrison-Kimmel et al. (2014a) with $2^{3}$ more particles (particle mass $2.4 \times 10^{4} \mathrm{M}_{\odot}$ ) show that simulations at our resolution converge for subhaloes above $V_{\max } \gtrsim 8 \mathrm{~km} \mathrm{~s}^{-1}$ and $R_{\max }$ resolved for $V_{\max } \gtrsim 15 \mathrm{~km} \mathrm{~s}^{-1}$. We identify haloes with ROCKSTAR $^{7}$ (Behroozi, Wechsler \& Wu 2013a), a publicly available six-dimensional friends-of-friends halo finder. Table 1 summarizes the physical properties of the main host halo in the zoom simulations. We define the virial mass $M_{\text {vir }}$ as mass contained within a sphere of radius $R_{\mathrm{vir}}$ that corresponds to an overdensity of 97 relative to the critical density of the Universe (Bryan \& Norman 1998). We define the $V_{\max }$ at the moment a halo has its maximum mass as $V_{\text {peak }}$. Extracting $V_{\text {peak }}$ thus needs the assembly of a merger tree, which we perform with CONSISTENT-TREES ${ }^{8}$ (Behroozi et al. 2013b).

A particular concern for WDM $N$-body simulations is the artificial fragmentation of filaments that cause the production of artificial clumps. These act as spurious haloes and contaminate the subhalo catalogues. Lovell et al. (2014) investigated the contamination based on a series of dissipationless WDM simulations. They adopted thermal WDM masses of $1.5,1.6,2.0$, and $2.3 \mathrm{keV}$, i.e. with cutoffs in the range of interest for our study, and find that the spurious subhaloes approximately equal the number of genuine subhaloes on scales of $\sim 10^{7} \mathrm{M}_{\odot}\left(\right.$ or $\left.V_{\max } \sim 5 \mathrm{~km} \mathrm{~s}^{-1}\right)$ for a $2.0 \mathrm{keV}$ WDM and MW-sized halo, and dominate on smaller scales. They test this using high-resolution (particle mass $1.55 \times 10^{4} \mathrm{M}_{\odot}$ ) and low-resolution (particle mass $4.43 \times 10^{5} \mathrm{M}_{\odot}$ ) analogues, i.e. our resolution falls in between their test cases. These suggest our resolved subhaloes (i.e. with $V_{\max }>8 \mathrm{~km} \mathrm{~s}^{-1}$ ) are not severely contaminated. This is also consistent with the scaling presented by Wang \& White (2007). However, the precise form of the subhalo mass function on scales smaller than the cutoff scale is uncertain (Hahn, Abel \& Kaehler 2013; Schneider et al. 2013). Instead of attempting to remove spurious subhaloes, we avoid the issue by only considering objects larger than $V_{\max }>8 \mathrm{~km} \mathrm{~s}^{-1}$.

\section{ANALYSES}

\subsection{Subhalo abundance}

We begin by exploring the abundance of dark matter substructures within the virial radius of the host halo. The three final columns of Table 1 show the total number of subhaloes within the virial radius with $V_{\max }>8 \mathrm{~km} \mathrm{~s}^{-1}, V_{\max }>15 \mathrm{~km} \mathrm{~s}^{-1}$, and $V_{\text {peak }}>20 \mathrm{~km} \mathrm{~s}^{-1}$ cuts, respectively. The cumulative distributions are shown in Fig. 3.

\footnotetext{
${ }^{7}$ https://code.google.com/p/rockstar/

${ }^{8} \mathrm{https} / / /$ bitbucket.org/pbehroozi/consistent-trees
}

They reveal a strong suppression in the number of subhaloes. The suppression in the number of low-mass subhaloes is consistent with the cutoff in the WDM transfer function. For example, s20.0 has the largest cutoff wavenumber (Fig. 2) and as a result shows the least suppression.

According to the standard paradigm, these subhaloes must host the satellites orbiting the main galaxy. Observationally, the number of satellites orbiting the MW currently stands at $\sim 28$, including 11 so-called classical dwarfs known pre-SDSS, a similar number of ultrafaint dSph galaxies discovered by the SDSS (Wolf et al. 2010; McConnachie 2012), and eight recently discovered candidates in the Dark Energy Survey (DES) fields (DES Collaboration 2015). We remove from this list Leo $\mathrm{T}$ and Eridanus II, which are both clearly further away than the virial radius of the MW. We keep the Large Magellanic Cloud (LMC) and the Small Magellanic Cloud (SMC), since the ELVIS haloes were intentionally chosen to contain realistic analogues to the Magellanic clouds (Garrison-Kimmel et al. 2014a). Our chosen halo has one subhalo with $V_{\max }>60 \mathrm{~km} \mathrm{~s}^{-1}$; had haloes been chosen at random, it would have been unlikely to find such massive subhaloes (Boylan-Kolchin et al. 2010). The satellite list should be taken as a lower limit, since ongoing and future more complete and deeper surveys are expected to lead to more discoveries. Indeed, six to eight candidate ultrafaint dSphs were recently reported in the DES field (Drlica-Wagner et al. 2015), and the intrinsic satellite count of the MW is estimated to be close to $\sim 100$ (Tollerud et al. 2008; Walsh et al. 2009; Hargis et al. 2014; Drlica-Wagner et al. 2015). Therefore, the simulations should contain at a minimum 28 subhaloes to host the known satellites, and certainly more.

The equivalent in M31 is aided by the Pan-Andromeda Archaeological Survey (PAndAS), which provides complete coverage out to $\sim 150 \mathrm{kpc}$ from M31 and sensitive to satellites down to luminosities of $\sim 10^{5} \mathrm{~L} \odot$ (Richardson et al. 2011). Larger distances have been subject to various studies, most recently the Panoramic Survey Telescope and Rapid Response System that provides a survey going deeper than SDSS and wider than PAndAS. The total number of satellites within $150 \mathrm{kpc}(300 \mathrm{kpc})$ is now 18 (35) (Conn et al. 2012; McConnachie 2012; Martin et al. 2013). While the total M31 satellite population is surprisingly similar to that of the MW, the MW count includes faint satellites $\left(L<10^{5} \mathrm{~L}_{\odot}\right)$ that are currently difficult to detect around M31. In fact, M31 contains significantly more bright satellites than the MW. As in the MW, therefore, current counts should be treated as a lower limit, particularly at the faint end.

To compare, we consider subhaloes with $V_{\text {peak }}>20 \mathrm{~km} \mathrm{~s}^{-1}$, because subhaloes with lower $V_{\text {peak }}$ may not have formed stars due to suppressed gas accretion during the reionization epoch (Bullock et al. 2000; Sawala et al. 2015). This condition is also consistent 


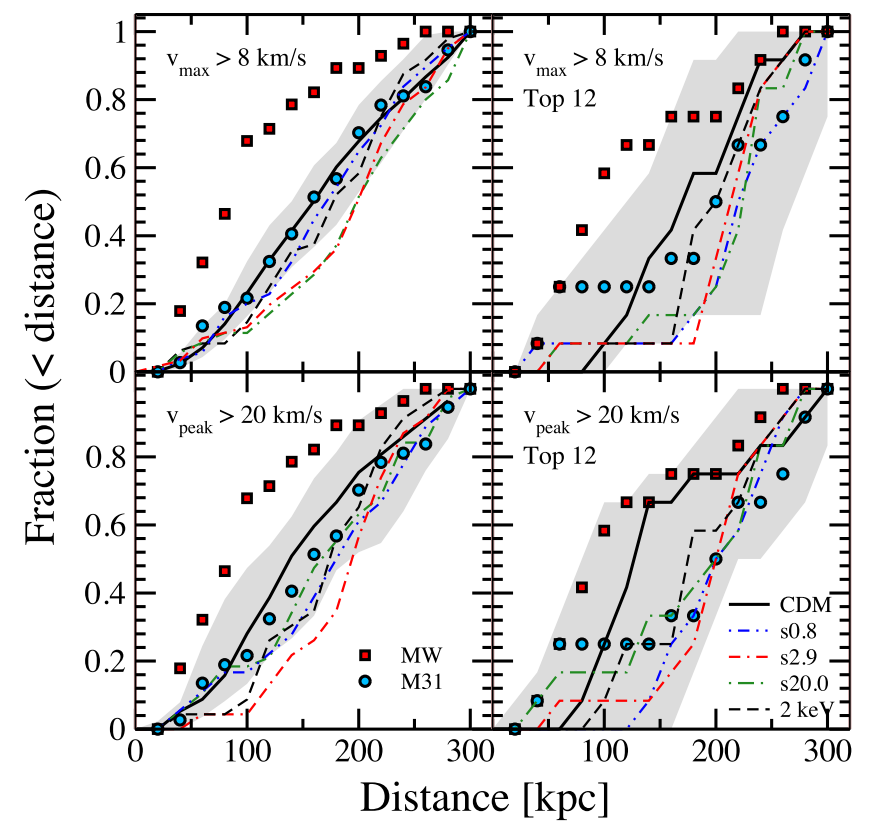

Figure 4. Normalized radial distributions of satellites for the MW and M31, and the same for subhaloes for five cosmologies: CDM (black solid), thermal $2.0 \mathrm{keV}$ WDM (black dashed), and three resonant sterile neutrino WDM s0.8 (blue double-dot-dashed), s2.9 (red dot-double-dashed), and s20.0 (green dot-dashed), as labelled. The grey band shows the spread in the CDM results based on the ELVIS suite of simulations. The four panels show: all satellites and all subhaloes with $V_{\max }>8 \mathrm{~km} \mathrm{~s}^{-1}$ (top left), all satellites and all subhaloes with $V_{\text {peak }}>20 \mathrm{~km} \mathrm{~s}^{-1}$ (bottom left), and their equivalents using only the most luminous 12 satellites and the largest 12 subhaloes (right-hand panels).

with extrapolation of abundance matching to MW satellite scales (Garrison-Kimmel et al. 2014b), in particular given the uncertainty in the scaling between stellar and halo masses (Garrison-Kimmel et al., in preparation). Comparing the final column in Table 1 to the known satellites, we see that s0.8 and s2.9 are disfavoured, while s20.0 satisfies the requirements. However, there is a factor of $\sim 2$ intrinsic spread from main halo selection (grey band in Fig. 3). We thus conclude that all the WDM simulations contain sufficient numbers of subhaloes to host the presently observed satellites, but the space for additional satellites is limited. We caution however that the constraint can be relaxed by considering galaxy formation in subhaloes with $V_{\text {peak }}<20 \mathrm{~km} \mathrm{~s}^{-1}$. For example, recent simulations suggest galaxy formation can occur in $V_{\text {peak }}<20 \mathrm{~km} \mathrm{~s}^{-1}$ subhaloes provided the process occurs at high enough redshifts (Wheeler et al. 2015).

\subsection{Radial profiles}

We next explore the radial distributions of the subhaloes and compare them to the observed satellites of the MW and M31. To make a comparison of the radial distribution shape, we normalize the distributions by the total number within the virial radius. The left-hand panels of Fig. 4 show the results when all resolved subhaloes are compared against the entire-known satellites population; the top and bottom panels show results applying a $V_{\max }>8 \mathrm{~km} \mathrm{~s}^{-1}$ cut and a $V_{\text {peak }}>20 \mathrm{~km} \mathrm{~s}^{-1}$ cut to the subhalo, respectively. In both cases, the simulations are in better agreement with the satellites of M31 than the satellites of the MW, mirroring analyses of the $\Lambda$ CDM ELVIS suite (Yniguez et al. 2014). The different cutoffs do not change this result. If additional satellites are discovered at large distances $(>150 \mathrm{kpc}$ ) around the MW, the radial distribution of MW satellites will more closely match those of simulated subhaloes and M31 satellites. On the other hand, there is presently a stronger luminosity bias in the M31 satellites, and future discoveries of dim satellite dwarfs may change the M31 curve.

The right-hand panels show the comparison when only the top 12 most massive subhaloes are considered: the top panel uses the 12 largest in $V_{\max }$, while the bottom panel uses the 12 largest in $V_{\text {peak }}$. These are compared against the most luminous 12 satellites which are likely to be observationally complete. For the MW, this is the LMC, SMC, Sagittarius, Fornax, Leo I, Sculptor, Carina, Leo II, Sextans, Ursa Minor, Draco, and Canes Venatici I; for M31, Triangulum (M33), And XXXI, NGC 205, M32, IC 1613, IC 10, NGC 185, NGC 147, And VII, And II, And I, and And VI. Due to the smaller numbers, the top 12 subhalo radial distribution shows considerably more scatter than the total. Within the scatter, the radial distributions of the 12 most massive subhaloes are marginally consistent with the brightest MW and M31 satellites.

In all comparisons, WDM realizations tend to yield radially expanded subhalo distributions compared to CDM. This is consistent with the later formation times of subhaloes in WDM than in CDM. The later formation times yield not only less-concentrated subhaloes, but they are also more susceptible to tidal disruption during near passages in the inner halo (e.g. Maccio' et al. 2010). Similar effects are seen in studies by Anderhalden et al. (2012) who explored the radial profiles of subhaloes in various WDM and CDM + WDM cosmologies. In general, this makes WDM realizations less compatible with the distribution of MW satellite radial positions, which are more concentrated. Currently, there is only a mild tension when limiting the comparison to the observationally complete luminous satellite sample (right-hand panels of Fig. 4). However, the radial position distribution will become a powerful diagnostic when a larger sample of complete satellite galaxies becomes available, e.g. from ongoing surveys such as DES, and future missions such as the Large Synoptic Survey Telescope and the Wide-Field Infrared Survey Telescope (Spergel et al. 2015) which will in particular be able to study satellite populations in nearby galaxies out to several Mpc.

We conclude that the radial position distributions of the subhaloes in our resonant sterile neutrino realizations are in agreement with the observed satellites of M31. While there remains some tension when compared with the satellites of the MW, this is significantly reduced when only the most luminous classical satellites are considered. Future satellite galaxy samples, both of MW and other galaxies, will help test this further.

\subsection{Internal structure of subhaloes}

We finally explore the internal structures of subhaloes. The simulations performed in this study do not fully resolve density profiles in the innermost $\sim 500 \mathrm{pc}$, but integral values such as $V_{\max }$ and $R_{\max }$ are converged for subhaloes with $V_{\max }>15 \mathrm{~km} \mathrm{~s}^{-1}$ (Garrison-Kimmel et al. 2014a). With these parameters, the two-parameter NavarroFrenk-White (NFW; Navarro et al. 1997) density profile is fully defined,

$\rho(r)=\frac{\rho_{s}}{\left(r / r_{s}\right)\left(1+r / r_{s}\right)^{2}}$,

where the characteristic scale radius $r_{s}=R_{\max } / 2.1626$ and the scale density $\rho_{s}=\rho_{s}\left(V_{\max }, R_{\max }\right)$. 


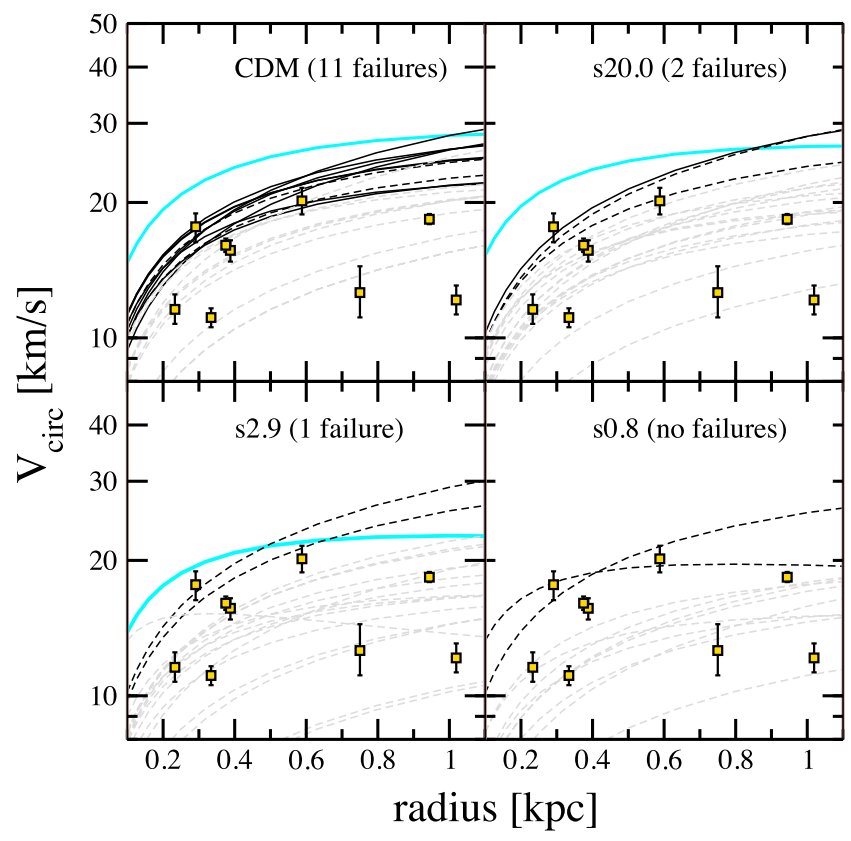

Figure 5. Rotation curves of all or 30 of the most massive subhaloes with $15<V_{\max } /\left(\mathrm{km} \mathrm{s}^{-1}\right)<60$. Values from measured circular velocities at halflight radii of the nine classical MW dwarfs, excluding LMC, SMC, and Sagittarius, are also plotted (symbols). Massive failures that cannot host any of the MW dSphs in the sample (thick solid cyan), additional massive failures from consideration of Draco and Ursa Minor (thin solid black), subhaloes that host either Draco or Ursa Minor (dashed black; only two allowed) and subhaloes that are consistent with at least one of the remaining seven dSphs in the sample (grey dashed), are shown.

By extrapolating the NFW profile, we determine the rotation curves of subhaloes, as shown in Fig. 5. Following Boylan-Kolchin et al. (2011), we only include subhaloes with $15<V_{\max } /\left(\mathrm{km} \mathrm{s}^{-1}\right)<60$; the LMC, SMC, and Sagittarius are excluded for a consistent comparison. For $\Lambda \mathrm{CDM}$ (top left panel), we show only the 30 most massive subhaloes; for the others, we show all the subhaloes that satisfy the $V_{\max }$ cut. These are compared to the circular velocities of $\mathrm{dSphs}$ at their deprojected half-light radius $\left(r=r_{1 / 2}\right)$. At this radius, accurate dynamical mass estimates are obtained (Wolf et al. 2010); the vertical error bars are derived from the $1 \sigma$ uncertainty in the mass at the half-light radius.

We use the 'strong massive failure' definition from GarrisonKimmel et al. (2014b), which considers all subhaloes that cannot be assigned to host a dwarf galaxy as massive failures, with the caveat that the two densest dwarfs (Draco and Ursa Minor) can only be hosted by a single subhalo each. In $\Lambda \mathrm{CDM}$, the number of massive failures is typically $\sim 20$, but with a large scatter from 2 to over 40 , depending on the halo mass, cosmology, and other variables. Our chosen halo contains 11 massive failures in $\Lambda \mathrm{CDM}$. We find that with our specific resonantly produced sterile neutrino dark matter, the number of massive failures is greatly reduced to between none to two.

An implicit assumption we have made is that the density profiles of subhaloes do not deviate from NFW at small radii in a WDM cosmology. This is not valid for radii smaller than some critical value, since WDM predicts the formation of a core beyond a WDM model-dependent density. However, the scales for core formation is less than $O(10)$ pc (Villaescusa-Navarro \& Dalal 2011; Macciò et al. 2012, 2013), i.e. not an important effect on scales of interest in this study.

\section{CONCLUSIONS}

In this paper, we have tested the near-field cosmological implications of a $7.1 \mathrm{keV}$ sterile neutrino as the origin of the anomalous $3.55 \mathrm{keV}$ lines recently discovered towards a number of massive dark matter objects. Specifically, we have simulated structure formation of a MW-size host from $z=125$ to $z=0$ using the latest primordial matter perturbation power spectrum implied by sterile neutrinos generated via the Shi-Fuller resonance mechanism, and explored the properties of their subhaloes: their number, radial distribution, and internal structure. We explored three sterile neutrino mixing angles, all consistent with the observed $3.55 \mathrm{keV}$ line and reproducing the cosmological dark matter abundance, but different in their power spectrum cutoff (Fig. 2). We also simulate a $2.0 \mathrm{keV}$ thermal WDM candidate for comparison purposes.

We find that a sterile neutrino responsible for the $3.55 \mathrm{keV}$ line implies non-trivial differences in the subhalo properties of a MWsized halo compared to CDM and thermal WDM. The number of subhaloes available to host satellite galaxies is reduced compared to CDM by factors of $\sim 2-3$ (Table 1). The suppressions are already significant: in some cases, the predicted number of subhaloes is already smaller than the number of observed satellites of the MW and M31. The sterile neutrino models are not ruled out however, once scatter is included, e.g. arising owing to the uncertain mass of the MW halo (Fig. 3). The distributions of the subhalo radial positions are systematically less concentrated than the observed satellite positions, although they are consistent when only the observationally complete luminous satellites and most massive subhaloes are considered (Fig. 4 bottom-right panel). Finally, the internal properties of the most massive subhaloes show a consistent reduction in the number of strong massive failures (Fig. 5).

The fact that the subhalo counts are very close to the number of satellites has two immediate implications. First, future discoveries of satellites will provide strong and direct tests of the resonantly produced sterile neutrino scenario. Indeed, the recent report of 6-8 candidate ultrafaint dwarf satellites (Drlica-Wagner et al. 2015) suggests a total of $\sim 100$ such objects should be found around the MW, consistent with theoretical predictions (Tollerud et al. 2008; Hargis et al. 2014). This would, e.g. be a factor $\sim 3$ larger than the resolved subhalo counts of the s0.8 scenario and put the s 0.8 interpretation of the $3.55 \mathrm{keV}$ in serious tension with observations.

Secondly, the closeness highlights the future importance of the details of the cutoff shape. In our example, the difference between s2.9 and $2.0 \mathrm{keV}$ thermal models are minimal for the most massive subhaloes $\left(V_{\max }>20 \mathrm{~km} \mathrm{~s}^{-1}\right)$, but the $2.0 \mathrm{keV}$ model gradually shows less subhaloes than s2.9 at smaller masses. The difference in the number of resolved $\left(V_{\max }>8 \mathrm{~km} \mathrm{~s}^{-1}\right)$ subhaloes is 13 , which is a 20-30 per cent effect. The smaller number of subhaloes appears consistent with the stronger cutoff in the $2.0 \mathrm{keV}$ model with respect to the s2.9 model (Fig. 2). However, the difference is most stark near the resolution limit, and another major concern is the degree of contamination of spurious haloes. These make it difficult to provide a robust quantitative statement. Nevertheless, our results indicate that studies of small-scale structures of sterile neutrino WDM is becoming sensitive to the details of the cutoff shape, and thus the exact resonant sterile neutrino cutoff should be used, rather than the approximate thermal equivalent. The impacts of the cutoff will be investigated, in a companion work, in the context of a wider range of transfer function shapes both in the Local Group and in representative volumes of the Universe at high redshift (Bozek et al. 2015). 


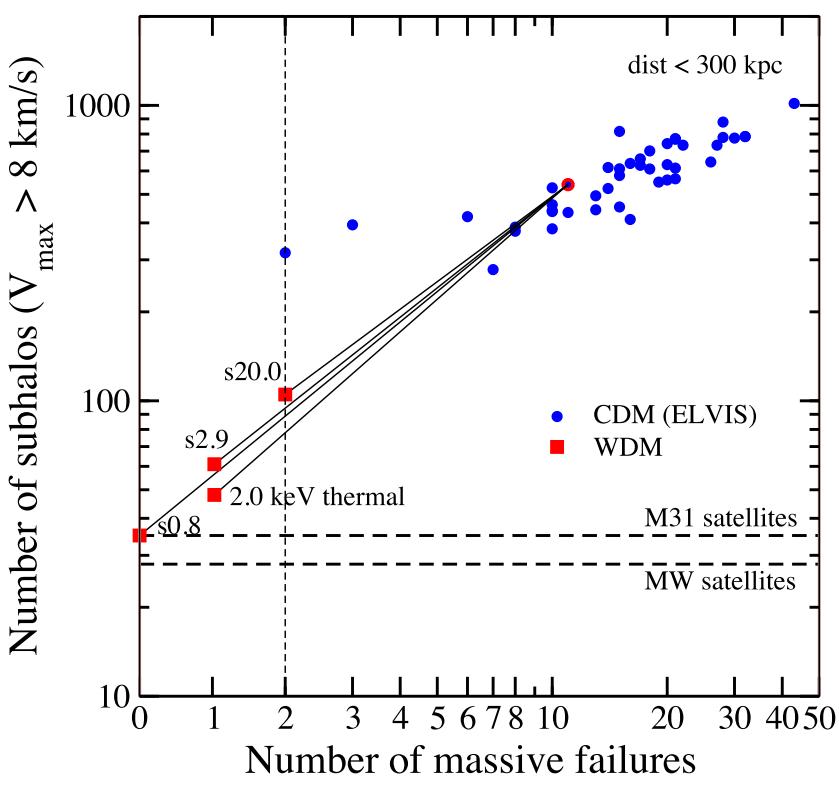

Figure 6. Summary of CDM and WDM zoom-in simulation results, showing the number of all subhaloes within $300 \mathrm{kpc}$ of the host and with $V_{\max }>8 \mathrm{~km} \mathrm{~s}^{-1}$ ( $y$-axis), and the number of massive failures ( $x$-axis; note the axis transitions from linear to logarithmic scale at 2 massive failures indicated by the vertical dashed line). Blue circles indicate CDM ELVIS simulations, and the halo selected to run in WDM highlighted by a red circle. The red squares indicate WDM with differing WDM particles, as labelled.

Fig. 6 shows a summary of our findings, plotting the number of subhaloes within $300 \mathrm{kpc}$ and with $V_{\max }>8 \mathrm{~km} \mathrm{~s}^{-1}$ on the $y$-axis, and the number of strong massive failures (based on $15<V_{\max } /\left(\mathrm{km} \mathrm{s}^{-1}\right)<60$ subhaloes $)$ on the $x$-axis. The blue circles denote the results based on the $\Lambda$ CDM simulations of the ELVIS suite, with the specific halo simulated in WDM shown with a red perimeter. The red squares are our WDM simulations, both the resonantly produced sterile neutrinos and thermal WDM, as labelled. The observed satellite counts of the MW and M31 are shown by dashed lines and labelled. The missing satellites problem is illustrated by the $y$-axis: the CDM simulations (blue circles) all produce far too many subhaloes relative to observed satellite galaxy counts (dashed horizontal lines). The $x$-axis represents TBTF: the CDM simulations predict a large population of dense subhaloes that cannot host the known MW dSphs. Sterile neutrino dark matter is effective at reducing the severity of the TBTF problem while matching the subhalo abundance. Overall, we conclude that a $7.1 \mathrm{keV}$ sterile neutrino provides a good description of the Local Group, which is often better than CDM in dissipationless simulations, but will be tested by future searches for MW and M31 satellites.

\section{ACKNOWLEDGEMENTS}

We thank Tejaswi Venumadhav and Francis-Yan Cyr-Racine for discussions and for providing digitized data of sterile neutrino transfer functions. SH and KNA acknowledge support from the Institute for Nuclear Theory programme 'Neutrino Astrophysics and Fundamental Properties' 15-2a where part of this work was done. KNA is partially supported by NSF CAREER Grant No. PHY-1159224 and NSF Grant No. PHY-1316792. MBK acknowledges support provided by NASA through HST theory grants (programs AR-12836 and AR-13888) from the Space Telescope Science Institute (STScI), which is operated by the Association of Universities for Research in Astronomy (AURA), Inc., under NASA contract NAS5-26555. Support for SGK. was provided by NASA through Einstein Postdoctoral Fellowship grant number PF5-160136 awarded by the Chandra $\mathrm{X}$-ray Center, which is operated by the Smithsonian Astrophysical Observatory for NASA under contract NAS8-03060.

\section{REFERENCES}

Abazajian K. N., 2014, Phys. Rev. Lett., 112, 161303

Abazajian K., Fuller G. M., Patel M., 2001a, Phys. Rev. D, 64, 023501

Abazajian K., Fuller G. M., Tucker W. H., 2001b, ApJ, 562, 593

Amorisco N. C., Zavala J., de Boer T. J. L., 2014, ApJ, 782, L39

Anderhalden D., Diemand J., Bertone G., Macciò A. V., Schneider A., 2012,

J. Cosmol. Astropart. Phys., 10, 47

Anderhalden D., Schneider A., Macciò A. V., Diemand J., Bertone G., 2013, J. Cosmol. Astropart. Phys., 3, 14

Anderson M. E., Churazov E., Bregman J. N., 2015, MNRAS, 452, 3905

Arraki K. S., Klypin A., More S., Trujillo-Gomez S., 2014, MNRAS, 438, 1466

DES Collaboration, 2015, ApJ, 807, 50

Behroozi P. S., Wechsler R. H., Wu H.-Y., 2013a, ApJ, 762, 109

Behroozi P. S., Wechsler R. H., Wu H.-Y., Busha M. T., Klypin A. A., Primack J. R., 2013b, ApJ, 763, 18

Belokurov V. et al., 2007, ApJ, 654, 897

Bode P., Ostriker J. P., Turok N., 2001, ApJ, 556, 93

Boyarsky A., Ruchayskiy O., Iakubovskyi D., Franse J., 2014a, Phys. Rev. Lett., 113, 251301

Boyarsky A., Franse J., Iakubovskyi D., Ruchayskiy O., 2014b, Phys. Rev. Lett., 115, 161301

Boylan-Kolchin M., Springel V., White S. D. M., Jenkins A., 2010, MNRAS, 406, 896

Boylan-Kolchin M., Bullock J. S., Kaplinghat M., 2011, MNRAS, 415, L40 Boylan-Kolchin M., Bullock J. S., Kaplinghat M., 2012, MNRAS, 422, 1203 Bozek B., Boylan-Kolchin M., Horiuchi S., Garrison-Kimmel S., Abazajian K., Bullock J. S., 2015, preprint (arXiv:1512.04544)

Brook C. B., Di Cintio A., 2015, MNRAS, 450, 3920

Brooks A. M., Zolotov A., 2014, ApJ, 786, 87

Bryan G. L., Norman M. L., 1998, ApJ, 495, 80

Bulbul E., Markevitch M., Foster A. R., Smith R. K., Loewenstein M., Randall S. W., 2014a, preprint (arXiv:1409.4143)

Bulbul E., Markevitch M., Foster A., Smith R. K., Loewenstein M., Randall S. W., 2014b, ApJ, 789, 13

Bullock J. S., Kravtsov A. V., Weinberg D. H., 2000, ApJ, 539, 517

Busha M. T., Alvarez M. A., Wechsler R. H., Abel T., Strigari L. E., 2010, ApJ, 710, 408

Conn A. R. et al., 2012, ApJ, 758, 11

Del Popolo A., Pace F., 2015, preprint (arXiv:1502.01947)

Del Popolo A., Lima J. A. S., Fabris J. C., Rodrigues D. C., 2014, J. Cosmol. Astropart. Phys., 4, 21

Di Cintio A., Brook C. B., Dutton A. A., Macciò A. V., Stinson G. S., Knebe A., 2014, MNRAS, 441, 2986

Donato F. et al., 2009, MNRAS, 397, 1169

The DES Collaboration, 2015, ApJ, 813, 109

Elbert O. D., Bullock J. S., Garrison-Kimmel S., Rocha M., Oñorbe J., Peter A. H. G., 2015, MNRAS, 453, 29

Ferrero I., Abadi M. G., Navarro J. F., Sales L. V., Gurovich S., 2012, MNRAS, 425, 2817

Flores R. A., Primack J. R., 1994, ApJ, 427, L1

Garrison-Kimmel S., Rocha M., Boylan-Kolchin M., Bullock J. S., Lally J., 2013, MNRAS, 433, 3539

Garrison-Kimmel S., Boylan-Kolchin M., Bullock J. S., Lee K., 2014a, MNRAS, 438, 2578

Garrison-Kimmel S., Boylan-Kolchin M., Bullock J. S., Kirby E. N., 2014b, MNRAS, 444, 222

Garrison-Kimmel S., Horiuchi S., Abazajian K. N., Bullock J. S., Kaplinghat M., 2014c, MNRAS, 444, 961

Governato F. et al., 2012, MNRAS, 422, 1231 
Griffen B. F., Ji A. P., Dooley G. A., Gómez F. A., Vogelsberger M., O’Shea B. W., Frebel A., 2015, preprint (arXiv:1509.01255)

Gritschneder M., Lin D. N. C., 2013, ApJ, 765, 38

Hahn O., Abel T., 2011, MNRAS, 415, 2101

Hahn O., Abel T., Kaehler R., 2013, MNRAS, 434, 1171

Hargis J. R., Willman B., Peter A. H. G., 2014, ApJ, 795, L13

Hinshaw G. et al., 2013, ApJS, 208, 19

Ho S. et al., 2012, ApJ, 761, 14

Horiuchi S., Humphrey P. J., Oñorbe J., Abazajian K. N., Kaplinghat M., Garrison-Kimmel S., 2014, Phys. Rev. D, 89, 025017

Iakubovskyi D., Bulbul E., Foster A. R., Savchenko D., Sadova V., 2015, preprint (arXiv:1508.05186)

Jeltema T., Profumo S., 2014, preprint (arXiv:1411.1759)

Jeltema T., Profumo S., 2015, MNRAS, 450, 2143

Kamionkowski M., Liddle A. R., 2000, Phys. Rev. Lett., 84, 4525

Klypin A., Kravtsov A. V., Valenzuela O., Prada F., 1999, ApJ, 522, 82

Koposov S. E., Yoo J., Rix H.-W., Weinberg D. H., Macciò A. V., Escudé J. M., 2009, ApJ, 696, 2179

Koposov S. E., Belokurov V., Torrealba G., Evans N. W., 2015, ApJ, 805, 130

Kravtsov A. V., Gnedin O. Y., Klypin A. A., 2004, ApJ, 609, 482

Kusenko A., 2006, Phys. Rev. Lett., 97, 241301

Larson D. et al., 2011, ApJS, 192, 16

Lewis A., Challinor A., Lasenby A., 2000, ApJ, 538, 473

Lovell M. R. et al., 2012, MNRAS, 420, 2318

Lovell M. R., Frenk C. S., Eke V. R., Jenkins A., Gao L., Theuns T., 2014, MNRAS, 439, 300

Maccio' A. V., Kang X., Fontanot F., Somerville R. S., Koposov S. E., Monaco P., 2010, MNRAS, 402, 1995

Macciò A. V., Paduroiu S., Anderhalden D., Schneider A., Moore B., 2012, MNRAS, 424, 1105

Macciò A. V., Paduroiu S., Anderhalden D., Schneider A., Moore B., 2013, MNRAS, 428, 3715

McConnachie A. W., 2012, AJ, 144, 4

Malyshev D., Neronov A., Eckert D., 2014, Phys. Rev. D, 90, 103506

Marsh D. J. E., Pop A.-R., 2015, MNRAS, 451, 2479

Martin N. F. et al., 2013, ApJ, 772, 15

Moore B., Ghigna S., Governato F., Lake G., Quinn T., Stadel J., Tozzi P., 1999, ApJ, 524, L19

Navarro J. F., Frenk C. S., White S. D. M., 1997, ApJ, 490, 493

Oh S.-H., de Blok W. J. G., Brinks E., Walter F., Kennicutt R. C., Jr, 2011, AJ, 141, 193

Okamoto T., Frenk C. S., 2009, MNRAS, 399, L174

Oñorbe J., Garrison-Kimmel S., Maller A. H., Bullock J. S., Rocha M., Hahn O., 2014, MNRAS, 437, 1894

Oñorbe J., Boylan-Kolchin M., Bullock J. S., Hopkins P. F., Kerěs D., Faucher-Giguère C.-A., Quataert E., Murray N., 2015, MNRAS, 454, 2092

Papastergis E., Giovanelli R., Haynes M. P., Shankar F., 2015, A\&A, 574, A113
Peñarrubia J., Pontzen A., Walker M. G., Koposov S. E., 2012, ApJ, 759, L42

Planck Collaboration XXIV, 2015, A\&A, 580, A22

Polisensky E., Ricotti M., 2014, MNRAS, 437, 2922

Pontzen A., Governato F., 2012, MNRAS, 421, 3464

Reed D. S., Schneider A., Smith R. E., Potter D., Stadel J., Moore B., 2015, MNRAS, 451, 4413

Richardson J. C. et al., 2011, ApJ, 732, 76

Ricotti M., Gnedin N. Y., 2005, ApJ, 629, 259

Riemer-Sorensen S., 2014, preprint (arXiv:1405.7943)

Rocha M., Peter A. H. G., Bullock J. S., Kaplinghat M., Garrison-Kimmel S., Oñorbe J., Moustakas L. A., 2013, MNRAS, 430, 81

Sawala T. et al., 2015, MNRAS, 448, 2941

Schneider A., Smith R. E., Reed D., 2013, MNRAS, 433, 1573

Schneider A., Anderhalden D., Macciò A. V., Diemand J., 2014, MNRAS, 441, L6

Shaposhnikov M., Tkachev I., 2006, Phys. Lett. B, 639, 414

Shi X., Fuller G. M., 1999, Phys. Rev. Lett., 82, 2832

Simon J. D., Bolatto A. D., Leroy A., Blitz L., Gates E. L., 2005, ApJ, 621, 757

Simpson C. M., Bryan G. L., Hummels C., Ostriker J. P., 2015, ApJ, 809, 69

Spergel D. et al., 2015, preprint (arXiv:1503.03757)

Springel V., 2005, MNRAS, 364, 1105

Tamura T., Iizuka R., Maeda Y., Mitsuda K., Yamasaki N. Y., 2015, PASJ, 67,23

Tollerud E. J., Bullock J. S., Strigari L. E., Willman B., 2008, ApJ, 688, 277

Urban O., Werner N., Allen S. W., Simionescu A., Kaastra J. S., Strigari L. E., 2015, MNRAS, 451, 2447

Venumadhav T., Cyr-Racine F.-Y., Abazajian K. N., Hirata C. M., 2015, preprint (arXiv:1507.06655)

Villaescusa-Navarro F., Dalal N., 2011, J. Cosmol. Astropart. Phys., 3, 24

Vogelsberger M., Zavala J., Loeb A., 2012, MNRAS, 423, 3740

Vogelsberger M., Zavala J., Simpson C., Jenkins A., 2014, MNRAS, 444, 3684

Walsh S. M., Willman B., Jerjen H., 2009, AJ, 137, 450

Wang J., White S. D. M., 2007, MNRAS, 380, 93

Wang M.-Y., Peter A. H. G., Strigari L. E., Zentner A. R., Arant B., GarrisonKimmel S., Rocha M., 2014, MNRAS, 445, 614

Wheeler C., Oñorbe J., Bullock J. S., Boylan-Kolchin M., Elbert O. D., Garrison-Kimmel S., Hopkins P. F., Kereš D., 2015, MNRAS, 453, 1305

Wolf J., Martinez G. D., Bullock J. S., Kaplinghat M., Geha M., Muñoz R. R., Simon J. D., Avedo F. F., 2010, MNRAS, 406, 1220

Yniguez B., Garrison-Kimmel S., Boylan-Kolchin M., Bullock J. S., 2014, MNRAS, 439, 73

Zentner A. R., Bullock J. S., 2002, Phys. Rev. D, 66, 043003

Zolotov A. et al., 2012, ApJ, 761, 71

This paper has been typeset from a $\mathrm{T}_{\mathrm{E}} \mathrm{X} / \mathrm{LT} \mathrm{E} \mathrm{X}$ file prepared by the author. 